ISSN: 2386-3919 - e-ISSN: 2386-3927

DOI: https://doi.org/10.14201/et20173512137

\title{
INFLUENCIA DEL CLIMA MOTIVACIONAL EN CLASE SOBRE EL COMPROMISO ESCOLAR DE LOS ADOLESCENTES Y SU LOGRO ACADÉMICO
}

\section{Influence of the motivational class climate on adolescents' school engagement and their academic achievement}

\author{
Melchor GuTiÉRReZ*, José Manuel TOMÁs**, José Marcos BARRICA**** \\ e Isabel ROMERO******* \\ * Universitat de Valencia. Correo-e: Melchor.Gutierrez@uv.es \\ ** Universitat de València. Correo-e: tomasjm@uv.es \\ ** Departamento de Ciências da Educação do Instituto Superior de Ciências da \\ Educação (Angola). Correo-e: barricajm@gmail.com \\ **** Universidade Katyavala Bwila (Angola).Correo-e: romeroisabel4@gmail.com
}

Recibido: 25/01/2016; Aceptado: 15/02/2017; Publicado: 31/05/2017

Ref. Bibl. MELCHOR GUTIÉRREZ, JOSÉ MANUEL TOMÁS, JOSÉ MARCOS BARRICA e ISABEL ROMERO. Influencia del clima motivacional en clase sobre el compromiso escolar de los adolescentes y su logro académico. Enseñanza \& Teaching, 35, 1-2017, 21-37.

RESUMEN: La literatura científica ofrece evidencia empírica de la relación entre el compromiso escolar y numerosas variables del contexto educativo de los adolescentes. El compromiso se ha relacionado, entre otros importantes constructos, con el burnout, tanto de los alumnos como de los profesores; el rendimiento escolar; la satisfacción con la escuela; los problemas de disciplina; las orientaciones de meta de los alumnos, y el clima motivacional de la clase. Por ello, el objetivo del presente trabajo ha sido analizar las relaciones entre el clima motivacional percibido en clase y el logro académico, mediado por el compromiso escolar de los alumnos. Han participado 2028 adolescentes que completaron diversos instrumentos para medir la percepción del clima motivacional de la clase, la satisfacción de las necesidades psicológicas básicas, el apoyo a la autonomía del alumno por el profesor y el logro 
MELCHOR GUTIÉRREZ, JOSÉ MANUEL TOMÁS, JOSÉ MARCOS BARRICA E ISABEL ROMERO INFLUENCIA DEL CLIMA MOTIVACIONAL EN CLASE SOBRE EL COMPROMISO ESCOLAR DE LOS ADOLESCENTES Y SU LOGRO ACADÉMICO

académico. Los datos se analizaron mediante modelos de ecuaciones estructurales con variables observables (path analysis). Los resultados mostraron una relación significativa entre el clima motivacional y el compromiso escolar, y de éste con el logro académico. Cabe destacar, además, la relación directa de la percepción de competencia y el apoyo a la autonomía con el éxito académico de los estudiantes. De las tres variables a predecir (Portugués, Matemáticas y Éxito académico), la mejor predicha por el clima de clase ha sido la percepción de éxito académico de los alumnos. Se discuten los resultados en el marco de la teoría de las metas de logro, de la motivación autodeterminada, y de cara a su aplicación práctica en el proceso de enseñanza-aprendizaje de los adolescentes.

Palabras clave: procesos educativos; compromiso del alumno; ambiente de clase; motivación del alumno; apoyo a la autonomía; logro académico.

SUMMARY: The scientific literature provides empirical evidence on the relationship between school engagement and numerous important variables of the adolescents' educational context. The school engagement has been related, among other important constructs, with burnout of both teachers and students, school performance, satisfaction with the school, behavioral disruption, goal orientation and motivational climate in the classroom. Because of it, the aim of this study was to analyze the relationship between perceived motivational class climate and students' academic achievement, with school engagement acting as a mediator. A sample of 2028 teenagers completed various instruments to measure the perception of motivational climate, perceived basic psychological needs satisfaction, perceived autonomy support provided by the teacher, and academic achievement. The data were analyzed using a structural equation model with observed variables (path analysis). The results have shown a significant relationship between motivational climate and school engagement, and of this with academic achievement. It should also be highlighted the direct relationship of perceived competence and perceived autonomy support with perception of academic success. Of the three variables to be predicted (Portuguese and Mathematics marks and Academic success), the largest percentage of variance explained was the one of academic success. The results are discussed within the framework of achievement goal theory, the self-determined motivation, and in terms of contributing practical issues to adolescents' teaching-learning process.

Key words: educational process; learner engagement; classroom environment; student motivation; autonomy support; academic achievement.

\section{INTRODUCCIÓN}

Los investigadores han venido generando una serie de conclusiones con respecto a los factores que se relacionan con el logro académico, en un intento por predecir el bajo rendimiento y prevenir el abandono escolar de los adolescentes (Mega, Ronconi y De Beni, 2014). Según datos de Eurostat (2013), por ejemplo en España, los índices de abandono escolar temprano son casi el doble que en la 
Unión Europea, aunque también es elevado en otras partes del mundo. Por ello, en el presente trabajo hemos analizado el papel que puede desempeñar el clima motivacional de la clase sobre el éxito académico de los adolescentes, mediado por su compromiso con la escuela.

El éxito académico es una de las variables más frecuentemente estudiadas cuando se analiza el desarrollo de los adolescentes (Jeynes, 2007; Winne y Nesbit, 2010). Está presente en numerosas investigaciones del contexto escolar, tanto en su vertiente de resultado positivo, justificando el desarrollo adecuado de los procesos educativos, como en su vertiente negativa, demandando la revisión de lo que no ha funcionado bien, cuando su nivel no es el esperado (Gordon, 2016). También se ha empleado como variable predictora del perfeccionismo (Damian, Stoeber, Negru y Baban, 2016) y como un indicador del ajuste escolar y del éxito futuro de los alumnos (Jelas, Azman, Zulnaidi y Ahmad, 2016; Xia, Fosco y Feinberg, 2016).

El éxito académico es un constructo que puede ser medido a través de diferentes indicadores. Aunque uno de los más utilizados es el rendimiento académico mediante las calificaciones escolares (Gordon, 2016; Noble y Sawyer, 2004; Reynolds et al., 2014), también se emplean otros indicadores, como la percepción que los alumnos tienen de su éxito en el desempeño de las tareas relacionadas con la escuela (Bandura, 1997; Damian et al., 2016; Plunkett, Henry, Houltberg, Sands y Abarca-Mortensen, 2008).

En las últimas décadas ha crecido el interés empírico por constructos psicoeducativos que pueden ser considerados la clave de los resultados de la educación (Green et al., 2012). Entre ellos se encuentran, por ejemplo, la motivación (Jang, Kim y Reeve, 2012; Pintrich, 2004; Wang y Holcombe, 2010) y el compromiso (Skinner, Kindermann y Furrer, 2009; Tomás, Gutiérrez, Sancho, Chireac y Romero, 2016).

El compromiso escolar tiene un carácter multidimensional (Christenson, Reschly y Wylie, 2012) y se define en relación con la implicación de los estudiantes para conseguir el logro académico. Aunque no existe acuerdo unánime entre los investigadores en cuanto a su composición, la concepción que actualmente predomina es la formada por cuatro factores: cognitivo, afectivo, conductual y de agencia personal. La dimensión cognitiva comprende el uso de estrategias de autorregulación como la memorización, el autocontrol o la planificación de tareas utilizadas en las actividades de aprendizaje (Reeve, 2012). La dimensión afectiva o emocional se refiere a la identificación de los alumnos con la escuela, las actitudes hacia el ámbito escolar, el aprendizaje, sus relaciones con los profesores, compañeros y otros profesionales del centro educativo. La dimensión conductual o comportamental se basa en las interacciones activas del alumno con su entorno académico, dirigidas a metas constructivas y persistentes (Fredricks, Blumenfeld y Paris, 2004). Incluye prestar atención en clase, invertir esfuerzo, participar en las discusiones y persistir en las tareas (Froiland y Oros, 2014; Reeve, Jang, Carrell, Jeon y Barch, 2004). Finalmente, el componente de agencia personal se define como la habilidad del alumno para establecer metas propias, constructivas, en el proceso activo del aprendizaje (Reeve y Tseng, 2011). 
En cuanto a la motivación, se han propuesto diferentes teorías para tratar de comprender cómo y por qué los estudiantes están motivados para el logro académico (Pintrich, 2004). De ellas, destacan la Teoría de Metas de Logro-TML (Ames, 1992; Nicholls, 1989) y la Teoría de la Autodeterminación-TAD (Deci y Ryan, 1985, 2008; Ryan y Deci, 2009). Ambas teorías son útiles para comprender los factores interpersonales y de motivación que ayudan a explicar las autoevaluaciones y el comportamiento de los alumnos (Linnenbrink y Pintrich, 2002).

Un concepto importante en la TML es el clima motivacional creado por los otros significativos (Ames, 1992; Nicholls, 1989). Esta teoría distingue entre climas de maestría y climas de rendimiento (Senko, Hulleman y Harackiewicz, 2011). Los climas se refieren a cómo los otros significativos (profesores, entrenadores, padres) estructuran el ambiente de aprendizaje. El clima de maestría se caracteriza por la promoción de aprendizaje y apoyo de los profesores y la percepción de los alumnos de una atmósfera de cooperación y ayuda donde el esfuerzo es importante para mejorar. Por el contrario, el clima de rendimiento se caracteriza por la promoción de competición por parte de los profesores, la comparación normativa de los estudiantes y la rivalidad entre ellos, así como la perspectiva de que los errores son penalizados (Senko et al., 2011).

La TAD contempla diferentes tipos de motivación que varían según sus niveles de autodeterminación a lo largo de un continuo. En el extremo más autodeterminado está la motivación intrínseca (cuando se realiza una actividad porque es placentera, divertida e interesante en sí misma para quien la hace) y en el extremo menos autodeterminado se encuentra la motivación extrínseca (cuando se realiza una actividad por obtener una recompensa o evitar un castigo). Por otro lado, la TAD postula que los seres humanos necesitan satisfacer tres necesidades psicológicas básicas: autonomía, competencia y relación. La autonomía se fundamenta en sentimientos de acción personal guiados por la voluntad propia, elección sin imposiciones; la competencia se caracteriza por sentimientos de capacidad para realizar las tareas a las que debe enfrentarse; y la relación hace referencia a sentimientos de conexión con los demás (Ryan y Deci, 2000). Las tres son importantes en el contexto educativo y las tres pueden ser promovidas por los profesores en el desarrollo de sus clases, creando climas facilitadores del desarrollo de los adolescentes (Cox y Williams, 2008). En este sentido, los profesores que se basan en un estilo de apoyo a la autonomía generalmente revitalizan la motivación de los estudiantes en términos de satisfacción de las necesidades psicológicas, mientras que los profesores que se apoyan en un estilo de control generalmente olvidan o incluso llegan a frustrar la motivación de los estudiantes y el funcionamiento de la clase (Raufelder, Regner, Drury y Eid, 2016; Reeve, 2009).

Los investigadores afirman que la motivación es un componente fundamental en cualquier modelo para comprender el logro académico, y que parece obvio que la motivación y el compromiso influyan conjuntamente sobre los resultados de los estudiantes. La motivación es un proceso no observable que conduce a un comportamiento observable que es el compromiso. Algunos estudios han analizado 
empíricamente estas relaciones, con hallazgos emergentes que apuntan hacia el valor predictivo de la motivación sobre el compromiso y, a su vez, de éste sobre los logros (Lau y Roeser, 2002; Lee, Hayes, Seitz, DiStefano y O'Connor, 2016).

Entre los estudios que han abordado el análisis de las relaciones de la motivación con el compromiso se encuentra, por ejemplo, el de Wang y Holcombe (2010), que desarrollaron una investigación longitudinal para analizar las relaciones entre las percepciones de los estudiantes del ambiente escolar, el compromiso escolar y el logro académico. Sus resultados apoyaron la concepción teórica de tres dimensiones diferentes, aunque relacionadas, del compromiso escolar: la participación escolar, el sentido de identificación con la escuela, y el uso de estrategias de autorregulación. Los resultados de este trabajo también indicaron que las percepciones del ambiente escolar por los estudiantes se relacionaron con el logro académico directamente, e indirectamente a través de los tres tipos de compromiso. Más específicamente, la percepción de los estudiantes de las características de la escuela durante el séptimo grado influyó en su participación escolar, su identificación con la escuela y el uso de estrategias autorreguladoras en octavo grado, lo cual, a su vez, influyó en el logro académico del octavo grado.

Con la misma intención se han desarrollado los trabajos de Froiland y Worrell (2016) y de Lee et al. (2016). En el primero de ellos, Froiland y Worrell (2016) han encontrado que la motivación intrínseca muestra un efecto positivo indirecto sobre el rendimiento, a través del compromiso escolar, resultado coincidente con los de otros trabajos de investigación realizados en el marco de la teoría de la autodeterminación, los cuales indican que la motivación conduce al compromiso (Ryan y Deci, 2009) y que el compromiso es el mediador clave entre la motivación y el rendimiento académico (Skinner et al., 2009). Por su parte, Lee et al. (2016) sometieron a verificación un modelo con el compromiso escolar como mediador de las relaciones entre varios constructos motivacionales y el logro de los estudiantes y encontraron que, así como el compromiso medió la relación entre la motivación intrínseca y el logro, no fue así para la motivación extrínseca. Parece ser que este tipo de motivación, la extrínseca, no muestra carácter predictivo del compromiso de los estudiantes y su efecto sobre el logro académico.

Aunque existen trabajos que guardan algunas similitudes con el que aquí presentamos, éste es el primer trabajo que considera el clima motivacional de la clase formado conjuntamente por el clima creado por los profesores, la percepción de los alumnos de la satisfacción de las necesidades psicológicas básicas y la percepción del apoyo del profesor a la autonomía del alumno. Fundamentados en los anteriores marcos teóricos, el objetivo de este trabajo ha consistido en analizar las relaciones entre el clima percibido en clase (clima motivacional, satisfacción de las necesidades psicológicas básicas y percepción de apoyo a la autonomía) y el logro académico de los alumnos (éxito académico, Portugués y Matemáticas), actuando como mediadores los cuatro factores del compromiso escolar de los estudiantes (Fig. 1). Se partió de varias hipótesis: (a) que el clima motivacional generado por los profesores en clase estaría positivamente relacionado con el 
MELCHOR GUTIÉRREZ, JOSÉ MANUEL TOMÁS, JOSÉ MARCOS BARRICA E ISABEL ROMERO INFLUENCIA DEL CLIMA MOTIVACIONAL EN CLASE SOBRE EL COMPROMISO ESCOLAR DE LOS ADOLESCENTES Y SU LOGRO ACADÉMICO

compromiso escolar de los estudiantes; (b) que la satisfacción de las necesidades psicológicas básicas estaría positivamente relacionada con el compromiso escolar; (c) que el apoyo a la autonomía del alumno por el profesor estaría positivamente relacionado con el compromiso de los estudiantes; y (d) que el compromiso escolar sería capaz de predecir el éxito académico de los adolescentes.

FIGURA 1

Modelo hipotético de las relaciones entre el clima motivacional en el aula, el compromiso escolar y el logro académico de los estudiantes

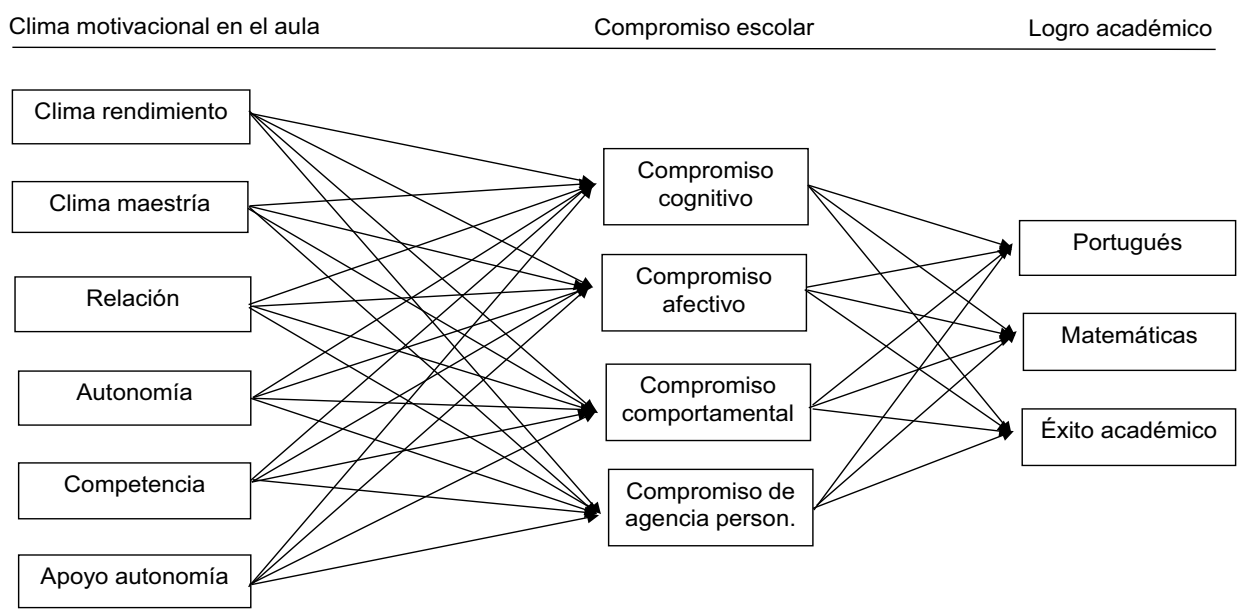

NOTA: Las flechas de doble dirección que denotan relaciones entre variables exógenas se han eliminado por simplicidad.

\section{MÉTODO}

\subsection{Participantes}

Participaron 2028 estudiantes de educación secundaria de la región de Benguela (Angola). Su edad media fue 17.5 años $(D T=2.31)$. El 50.1\% eran mujeres. El 52.8\% vivían en áreas urbanas. La encuesta, autoadministrada, se realizó en sus aulas y en el horario escolar habitual. Los encuestadores estaban entrenados para resolver cualquier duda o problema que los alumnos pudieran plantear. El tiempo invertido en completar la encuesta fue aproximadamente de 15 minutos.

\subsection{Instrumentos}

Todos los instrumentos aplicados a la muestra de estudio estaban formulados para que los alumnos respondiesen en una escala tipo Likert de 5 anclajes, desde (1) Totalmente en desacuerdo hasta (5) Totalmente de acuerdo. 
MELCHOR GUTIÉRREZ, JOSÉ MANUEL TOMÁS, JOSÉ MARCOS BARRICA E ISABEL ROMERO

INFLUENCIA DEL CLIMA MOTIVACIONAL EN CLASE SOBRE EL COMPROMISO ESCOLAR DE LOS ADOLESCENTES Y SU LOGRO ACADÉMICO

- Clima motivacional en el aula. Para medir la percepción de los alumnos sobre el clima motivacional que reinaba en sus clases, se emplearon tres instrumentos:

(i) Cuestionario de orientación y clima motivacional (Motivational Orientation and Climate Scale-MOC; Stornes y Bru, 2011). Para los objetivos de este trabajo se utilizaron únicamente los dos factores de clima motivacional generado por el profesor, que miden el clima de maestría o aprendizaje, con una fiabilidad de .72 , y el clima de rendimiento o comparación, con una fiabilidad de .75 .

(ii) Escala de satisfacción de las necesidades psicológicas básicas (Balanced Measure of Psychological Needs-BMPN; Sheldon y Hilper, 2012). Está compuesto por 18 ítems y mide tres necesidades psicológicas básicas: autonomía, competencia y relación. La fiabilidad de la escala fue de $\alpha$ $=.70$.

(iii) Cuestionario de percepción de apoyo a la autonomía del alumno (Perceived Autonomy Support; Jang et al., 2012). Formado por seis ítems que se agrupan en una sola dimensión. El alfa para esta muestra fue de .78 .

- Compromiso escolar. Para evaluar el compromiso de los estudiantes con la escuela se empleó la School Engagement Scale-SES-4D (Veiga, 2013), compuesta por un total de 20 ítems, cinco por cada uno de los cuatro factores: cognitivo, afectivo, comportamental y de agencia personal. La fiabilidad para esta muestra fue de $\alpha=.71$.

- Logro académico. El logro académico se midió a través de dos indicadores:

(i) Cuestionario de éxito académico (Academic Success; Plunkett et al., 2008). Consta de cinco ítems agrupados en un solo factor, con $\alpha=.82$.

(ii) Calificaciones escolares. Se contemplaron las calificaciones que los alumnos habían obtenido en Portugués y Matemáticas el último año académico, reflejadas en una escala de 0 a 20.

\subsection{Procedimiento}

Tras recibir del Ministerio de Educación de Angola el permiso para llevar a cabo la investigación, se contactó con las autoridades de los centros educativos de cuyos estudiantes se iba a obtener la información, para explicarles en qué consistía la investigación y solicitarles su colaboración. Seguidamente se habló con los alumnos, se les explicaron los instrumentos y se les invitó a participar. Se les facilitó una carta dirigida a sus padres o tutores (a quienes eran menores de edad) solicitando su autorización para la participación en el estudio. Todos los alumnos que participaron lo hicieron voluntariamente, tras devolver el consentimiento firmado de sus 
MELCHOR GUTIÉRREZ, JOSÉ MANUEL TOMÁS, JOSÉ MARCOS BARRICA E ISABEL ROMERO INFLUENCIA DEL CLIMA MOTIVACIONAL EN CLASE SOBRE EL COMPROMISO ESCOLAR DE LOS ADOLESCENTES Y SU LOGRO ACADÉMICO

padres o tutores. Previo a su aplicación, se procedió a traducir los instrumentos al portugués, empleando el sistema de doble traducción (back-translation). La aplicación de los instrumentos la realizó un miembro del equipo de investigación, en el horario habitual de clase pero sin la presencia de los profesores, para garantizar la confidencialidad de las respuestas.

\subsection{Análisis estadísticos}

Los datos fueron tratados mediante Modelos de Ecuaciones Estructurales con variables observables (path analysis) de carácter predictivo, y con análisis de los efectos mediadores de las dimensiones del compromiso escolar, para predecir el logro académico a partir del clima motivacional y el apoyo a la autonomía en el aula. Los modelos confirmatorios se estimaron mediante máxima verosimilitud con correcciones robustas de Satorra-Bentler en los errores estándar y estadísticos e índices de ajuste (Finney y DiStefano, 2006). Para evaluar la bondad de ajuste de estos modelos se emplearon diversos estadísticos e índices. En concreto, los recomendados en la literatura, pertenecientes a diversas familias: el estadístico de chi-cuadrado; el CFI (Comparative Fit Index); el RMSEA (Root Mean Squared Error of Approximation); y el GFI (Goodness of Fit Index; Kaplan, 2000). Se empleó el paquete estadístico EQS 6.7 (Bentler, 2006) para estimar todos los modelos estructurales. Valores del CFI y GFI por encima de .90 y mejor aún por encima de .95 se consideran ajustes excelentes, mientras que valores de RMSEA menores de .08 (y mejor por debajo de .05) también indican ajuste excelente (Hu y Bentler, 1999).

\section{Resultados}

\subsection{Correlaciones entre las variables estudiadas}

Tras haber comprobado que los instrumentos empleados para la recogida de datos eran adecuados para su aplicación a la presente muestra de estudio, se procedió a calcular las correlaciones que figuran en la Tabla 1. De todas ellas cabe señalar, por ejemplo, las correlaciones estadísticamente significativas entre la percepción del clima de maestría y la satisfacción de las tres necesidades básicas, mientras que las correlaciones de éstas con el clima de rendimiento son significativamente más bajas. 
MELCHOR GUTIÉRREZ, JOSÉ MANUEL TOMÁS, JOSÉ MARCOS BARRICA E ISABEL ROMERO

INFLUENCIA DEL CLIMA MOTIVACIONAL EN CLASE SOBRE EL COMPROMISO ESCOLAR DE LOS ADOLESCENTES Y SU LOGRO ACADÉMICO

TABLA 1

Correlaciones entre las variables estudiadas

\begin{tabular}{lcccccccccccc}
\hline \multicolumn{1}{c}{ VARIABLES } & 2 & 3 & 4 & 5 & 6 & 7 & 8 & 9 & 10 & 11 & 12 & 13 \\
\hline 1. Cl. Rend. & $.09^{* *}$ & $.05^{*}$ & $.12^{* *}$ & $.10^{* *}$ & $.13^{* * *}$ & $.10^{* *}$ & $.05^{*}$ & .03 & $.09^{* *}$ & .01 & .02 & .03 \\
2. Cl. Maestría & 1 & $.35^{* *}$ & $.28^{* *}$ & $.29^{* *}$ & $.43^{* * *}$ & $.26^{* *}$ & $.31^{* * *}$ & $.12^{* *}$ & $.21^{* *}$ & .04 & $.06^{*}$ & $.21^{* * *}$ \\
3. Relación & & 1 & $.30^{* *}$ & $.35^{* *}$ & $.35^{* *}$ & $.25^{* *}$ & $.28^{* *}$ & $.08^{* *}$ & $.27^{* *}$ & -.01 & -.01 & $.17^{* * *}$ \\
4. Autonomía & & & 1 & $.34^{* *}$ & $.31^{* *}$ & $.24^{* *}$ & $.23^{* *}$ & .02 & $.24^{* *}$ & .01 & $.08^{* * *}$ & $.17^{* * *}$ \\
5. Competenc. & & & 1 & $.39^{* * *}$ & $.31^{* *}$ & $.26^{* *}$ & $.09^{* *}$ & $.29^{* *}$ & $.09^{* * *}$ & $.11^{* * *}$ & $.19^{* * *}$ \\
6. Ap. Auton. & & & & 1 & $.35^{* *}$ & $.32^{* * *}$ & .02 & $.32^{* *}$ & .03 & $.08^{* * *}$ & $.18^{* * *}$ \\
7. C. Cognitivo & & & & & 1 & $.35^{* * *}$ & $.13^{* *}$ & $.46^{* *}$ & $.12^{* * *}$ & $.10^{* * *}$ & $.21^{* * *}$ \\
8. C. Afectivo & & & & & & 1 & $.15^{* * *}$ & $.29^{* *}$ & .02 & .01 & $.17^{* * *}$ \\
9. C. Conduct. & & & & & & & 1 & .01 & $.12^{* * *}$ & $.09^{* * *}$ & .04 \\
10. C. Ag. pers. & & & & & & & & 1 & .02 & .03 & $.15^{* * *}$ \\
11. Portugués & & & & & & & & & 1 & $.29^{* * *}$ & $.07^{* * *}$ \\
12. Matemátic. & & & & & & & & & & 1 & .02 \\
13. Éxito Acad. & & & & & & & & & & 1 \\
\hline
\end{tabular}

${ }^{*} \mathrm{P}<.05 ; * \mathrm{w}^{\mathrm{p}} \mathrm{p}<.01$

También son destacables las relaciones del clima de maestría y las necesidades básicas con la percepción de apoyo a la autonomía por el profesor. Por otro lado, cabe señalar las correlaciones entre las tres necesidades básicas y el compromiso escolar de los estudiantes, destacando principalmente los valores con el compromiso cognitivo, el afectivo y el de agencia personal, y siendo menos significativas las relaciones con el compromiso conductual. Dado que posteriormente se verán matizadas estas relaciones en el modelo estructural testado, finalizaremos resaltando las correlaciones positivas y estadísticamente significativas del clima de maestría, las necesidades básicas, el apoyo a la autonomía, el compromiso cognitivo, el compromiso afectivo y el de agencia personal con el éxito académico, siendo menores, y a veces inexistentes, estas relaciones con las calificaciones obtenidas tanto en Portugués como en Matemáticas. Por último, cabe señalar que el compromiso conductual se correlaciona de manera más significativa con las calificaciones (Portugués) que con la percepción de éxito académico.

\subsection{Predicción del logro académico de los adolescentes}

El modelo hipotético inicial (Fig. 1) planteaba que la percepción del clima motivacional de la clase (compuesto por el clima generado por los profesores, la satisfacción de las necesidades psicológicas básicas y el apoyo a la autonomía por el profesor) podría predecir de forma directa y positiva el compromiso de los estudiantes y éste, a su vez, sería capaz de predecir su logro académico. El modelo, por tanto, planteaba la mediación total del compromiso en la relación 
MELCHOR GUTIÉRREZ, JOSÉ MANUEL TOMÁS, JOSÉ MARCOS BARRICA E ISABEL ROMERO INFLUENCIA DEL CLIMA MOTIVACIONAL EN CLASE SOBRE EL COMPROMISO ESCOLAR DE LOS ADOLESCENTES Y SU LOGRO ACADÉMICO

motivación-rendimiento. Este modelo a-priori se puso a prueba mediante modelos de ecuaciones estructurales con variables observables para predecir el logro académico, a partir de la percepción del clima motivacional, actuando como mediador el compromiso escolar. Los índices de ajuste del modelo resultaron satisfactorios: $\chi_{(18)}^{2}=221.65, p<.001$, GFI $=.98$, CFI $=.94$, RMSEA $=.075$. No obstante, el test de Multiplicadores de Lagrange (LM test) ofreció información sobre dos importantes relaciones: los efectos directos de la competencia (como necesidad básica) y de la percepción de apoyo a la autonomía por el profesor sobre el éxito académico de los alumnos. Estos dos efectos directos no se hipotetizaban en el modelo original, pero son de relevancia teórica. Así pues, se añadieron estas relaciones al modelo inicial, con lo cual se mejoró el ajuste. Los índices de este segundo modelo fueron los siguientes: $\chi^{2}{ }_{(16)}=129.29, p<.001, \mathrm{GFI}=.99, \mathrm{CFI}=.97$, RMSEA $=.059$, lo cual muestra un excelente ajuste del modelo a los datos (Fig. 2).

Los resultados de este segundo modelo testado (Fig. 2) muestran que el clima generado por los profesores, la satisfacción de las necesidades psicológicas básicas y el apoyo a la autonomía por el profesor predicen el 18\% del compromiso cognitivo, el 17\% del compromiso afectivo, el $5 \%$ del compromiso conductual y el $16 \%$ del compromiso de agencia personal. Las variables del clima motivacional con mayor capacidad predictiva sobre el compromiso escolar han sido: el clima de rendimiento sobre el compromiso conductual $(\beta=.167, \mathrm{p}<.01)$; el clima de maestría sobre el compromiso afectivo y el conductual, con coeficientes estructurales de $\beta=.160$ y $\beta=.127(\mathrm{p}<.01)$, respectivamente; la necesidad de relación sobre el compromiso afectivo y el compromiso de agencia personal, con coeficientes estructurales de $\beta=.123$ y $\beta=.118$ ( $\mathrm{p}<.01$ ); la necesidad de competencia sobre el compromiso cognitivo y el compromiso de agencia personal, con coeficientes estructurales de $\beta=.135$ y $\beta=.141$ ( $p<.01)$; y la percepción de apoyo a la autonomía sobre el compromiso cognitivo, el compromiso afectivo y el compromiso de agencia personal, con coeficientes estructurales de $\beta=.202, \beta=.154$ y $\beta=.185(\mathrm{p}<.01)$. Por otro lado, el compromiso escolar predice significativamente el logro académico, aunque con valores relativamente bajos. Cabe señalar, así mismo, que el indicador del logro académico de los estudiantes mejor predicho ha sido el éxito académico, con un $21 \%$, habiendo sido las aportaciones más significativas para esta predicción las procedentes del compromiso cognitivo $(\beta=.178, \mathrm{p}<.01)$, del compromiso afectivo $(\beta=.140, \mathrm{p}<.01)$, y los efectos directos de la necesidad de competencia $(\beta=.169, \mathrm{p}<.01)$ y la percepción de apoyo por el profesor a la autonomía del alumno $(\beta=.162, \mathrm{p}<.01)$. 
MELCHOR GUTIÉRREZ, JOSÉ MANUEL TOMÁS, JOSÉ MARCOS BARRICA E ISABEL ROMERO

\section{FIGURA 2}

Solución estandarizada para el modelo propuesto de las relaciones entre el clima motivacional en el aula, el compromiso escolar y el logro académico de los adolescentes.

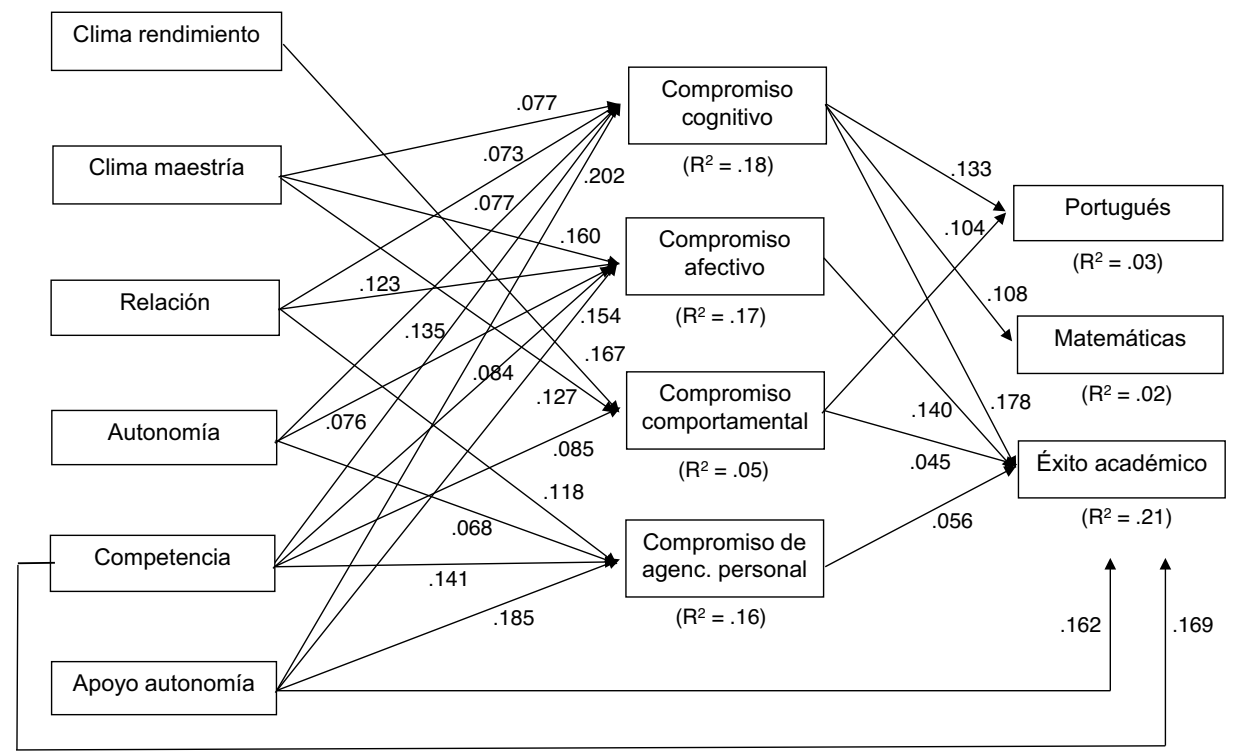

NOTAS: Todas las relaciones son estadísticamente significativas $(\mathrm{p}<.05)$; las flechas de doble dirección que denotan relaciones entre variables exógenas se han eliminado por simplicidad, pero pueden consultarse en la Tabla 1.

\section{DisCUSIÓN}

Como ha quedado anteriormente señalado, el compromiso escolar ha sido considerado por la investigación educativa una manifestación observable de la motivación de los alumnos hacia el aprendizaje, y algunos estudios han concluido que puede ser un buen predictor del logro académico. Tanto la teoría como la investigación sugieren que la motivación predice el compromiso y que éste, a su vez, predice el logro académico (Froiland y Oros, 2014; Greene, Miller, Crowson, Duke y Akey, 2004). Tal afirmación nos llevó a desarrollar el presente trabajo, con el objetivo de analizar la capacidad predictiva del clima motivacional de la clase sobre el logro académico de los alumnos, mediado por su compromiso con la escuela. Partimos de cuatro hipótesis, cuyo cumplimiento seguidamente iremos comentando a la luz de los resultados obtenidos.

En cuanto a la primera hipótesis, que planteaba que el clima motivacional creado por los profesores en clase estaría positivamente relacionado con el 
compromiso escolar de los estudiantes, los resultados mostrados en la Fig. 2 indican su cumplimiento en la mayoría de las relaciones hipotetizadas. Hay que matizar, no obstante, que así como el clima de maestría se relaciona con tres de las cuatro dimensiones del compromiso escolar (cognitivo, afectivo y comportamental), el clima de rendimiento únicamente se relaciona con el compromiso comportamental, no mostrando relación con los otros tres factores. Una vez más, el clima motivacional fundamentado en la maestría se muestra más significativo en los ambientes de aprendizaje que el clima de rendimiento o comparación, habitualmente con mayor presencia en contextos de logro más competitivos como pueden ser los ambientes deportivos. Dado que, por definición, el clima de maestría se asocia más directamente con la motivación intrínseca y el clima de rendimiento está más próximo a la motivación extrínseca, un trabajo que ayuda a interpretar nuestros resultados es el desarrollado por Lee et al. (2016), quienes argumentan que los motivadores extrínsecos pueden socavar la curiosidad, la persistencia y el interés de los estudiantes (Cerasoli, Nicklin y Ford, 2014; Deci, Koestner y Ryan, 2001), atributos que se asocian con el compromiso cognitivo, afectivo y conductual en las tareas de aprendizaje y en última instancia con el rendimiento escolar.

La segunda hipótesis, que planteaba que la satisfacción de las necesidades psicológicas básicas estaría positivamente relacionada con el compromiso escolar de los estudiantes, puede considerarse cumplida. Los resultados indican que tanto la satisfacción de la necesidad de relación como la necesidad de autonomía predicen el compromiso cognitivo, el afectivo y el de agencia personal. La satisfacción de la necesidad de autonomía es la de mayor capacidad predictiva, puesto que no sólo se muestra relacionada con los cuatro factores del compromiso escolar, sino que además también está directamente relacionada con el éxito académico. Estos resultados corroboran los obtenidos por Raufelder et al. (2014), que también encontraron que la necesidad de autonomía, relación y competencia se correlacionaban positivamente con el compromiso escolar emocional y el conductual, acorde tanto con Reeve et al. (2004) como con Ryan y Deci (2009). Nuestros resultados resaltan la importancia de la autodeterminación para las prácticas psicológicas y educativas, como también señalaron Niemiec y Ryan (2009). Estos resultados avalan la idea de que el apoyo de los profesores a la satisfacción de las necesidades psicológicas básicas de los estudiantes (autonomía, competencia y relación) no sólo facilita su autorregulación para el aprendizaje, el logro académico y el bienestar (Niemiec y Ryan, 2009), sino también su compromiso escolar.

Con respecto a la tercera hipótesis, según la cual el apoyo a la autonomía del alumno por el profesor estaría positivamente relacionado con el compromiso de los estudiantes, también puede considerarse cumplida, tras haberla encontrado relacionada con el compromiso cognitivo, el afectivo y el de agencia personal, además de presentar una relación directa con el éxito académico. Estos resultados son coincidentes con los obtenidos por Jang et al. (2012) y ponen de relieve que el apoyo a la autonomía proporcionado por el profesor mejora la participación de los estudiantes, como ya señalaron también Reeve, Jang, Carrell, Jeon y Barch (2004). 
En estudios anteriores se ha comprobado que el apoyo a la autonomía ejerce un efecto positivo para cada componente específico del compromiso, incluyendo el comportamental, emocional (Skinner, Furrer, Marchand y Kindermann, 2008), cognitivo (Vansteenkiste, Simons, Lens, Soenens y Matos, 2005) y de agencia personal (Reeve y Tseng, 2011). En este sentido, Reeve y Halusic (2009) argumentan que la razón por la cual el apoyo a la autonomía que proporciona el profesor facilita la participación se debe a que nutre la necesidad subyacente de los estudiantes por la autonomía, despertando en ellos una fuente de compromiso, lo cual supone el fomento de la motivación. Se ha comprobado que la percepción de apoyo a la autonomía es un predictor directo de la persistencia de los estudiantes en el desarrollo de sus tareas escolares (Vansteenkiste, Simons, Lens, Sheldon y Deci, 2004), la comprensión conceptual (Vansteenkiste et al., 2005) y la agencia personal (Reeve y Tseng, 2011).

Por último, la cuarta hipótesis planteaba que el compromiso escolar sería capaz de predecir el logro académico de los estudiantes. A la vista de los resultados obtenidos en este trabajo, esta hipótesis también se ha visto cumplida, aunque no con todas las relaciones hipotetizadas, ni con el mismo peso en todas las relaciones, siendo el compromiso cognitivo el que mayor predicción ha aportado al logro académico de los estudiantes. Estos resultados se encuentran en línea con los hallados en los estudios de Greene et al. (2004), de Froiland y Oros (2014) y de Lee et al. (2016).

Considerados en su conjunto, los resultados de este trabajo se encuentran en consonancia con los obtenidos por Green et al. (2012) y los de Martin, Collie, Mok y McInermey (2016), según los cuales la percepción que los alumnos tenían de las metas de la escuela estaba asociada con las metas personales, éstas se asociaron con el compromiso escolar y éste se asoció con el éxito académico. También encontraron efectos significativos indirectos de la percepción de las metas de la escuela sobre el logro académico a través del compromiso de los alumnos.

\subsection{Conclusión}

El clima motivacional de la clase contribuye al compromiso escolar, y lo hace con peso diferente de cada predictor, siendo el clima de maestría y la percepción de apoyo a la autonomía las variables de mayor aportación. A su vez, el compromiso escolar de los estudiantes contribuye a su logro académico, si bien hay que matizar que es la percepción de éxito académico (de carácter cognitivo) la variable mejor predicha, y no tanto las calificaciones en Portugués y en Matemáticas (variables cuantitativas y externas a la percepción del estudiante). Esto viene a sugerir que los profesores no sólo deben preocuparse por incrementar el compromiso escolar de sus alumnos, sino que deben comenzar por crear climas motivacionales en sus aulas principalmente orientados a la maestría, así como proporcionar apoyo a la autonomía de sus alumnos, porque de este modo promoverán un compromiso más profundo, lo cual revertirá en un mayor logro académico. 

DE LOS ADOLESCENTES Y SU LOGRO ACADÉMICO

\subsection{Limitaciones y prospectiva}

Este estudio presenta algunas limitaciones. La primera de ellas es que aunque el tamaño de la muestra es amplio, ésta se ha reclutado de manera incidental y a través de colaboradores (aunque se ha procurado un equilibrio en cuanto a distribución de edades, sexo y localización en la zona estudiada). También hay que tener en cuenta que los datos recogidos pertenecen a un solo momento temporal, lo cual impide establecer relaciones causales. Futuros estudios de carácter longitudinal podrían analizar el grado en que el clima motivacional y el compromiso contribuyen al desarrollo académico a largo plazo. Además, trabajos de intervención con los estudiantes podrían proporcionar una información más exacta de la contribución de cada variable en el incremento del logro académico. Otro aspecto a considerar es que los datos aquí recolectados han sido obtenidos mediante autoinformes. En trabajos futuros, variables como el compromiso escolar deberían ser evaluadas no sólo a partir de las opiniones de los alumnos, sino también a partir de informes de los profesores. Aunque las percepciones de los estudiantes son importantes, también deberían complementarse éstas con medidas más objetivas (o al menos externas al propio estudiante) del clima motivacional de la clase, así como medidas también más objetivas de su rendimiento académico, por ejemplo mediante pruebas de rendimiento preparadas al efecto. Finalmente, se sugiere, para futuras investigaciones, el empleo de la medida del compromiso conductual, con otra escala más en consonancia con la filosofía que de este factor presenta la literatura especializada, que no esté tan específicamente referida al mal comportamiento de los alumnos.

\section{REFERENCIAS BIBLIOGRÁFICAS}

Ames, C. (1992). Classrooms: goals, structures, and student motivation. Journal of Educational Psychology, 84, 261-271.

Bandura, A. (1997). Self-efficacy: The exercise of control. NewYork: Freeman.

Bentler, P. M. (2006). EQS 6. Structural Equations Program Manual. Encino, CA: Multivariate Software, Inc.

Cerasoli, C. P.; Nicklin, J. M. y Ford, M. T. (2014). Intrinsic motivation and extrinsic incentives jointly predict performance: A 40-year meta-analysis. Psychological Bulletin, 140 (4), 980-1008.

Christenson, S. L.; Reschly, A. L. y Wylie, C. (2012). Preface. En S. L. Christenson, A. L. Reschly y C. Wylie (Eds.). Handbook of research on student engagement. New York: Springer.

Cox, A. y Williams, L. (2008). The roles of perceived teacher support, motivational climate, and psychological needs satisfaction in students' physical education motivation. Journal of Sport and Exercise Psychology, 30, 222-239.

Damian, L. E.; Stoeber, J.; Negru, O. y Baban, A. (2016). On the development of perfectionism: the longitudinal role of academic achievement and academic efficacy. Journal of Personality. doi:10.1111/jopy.12261.

Deci, E. L.; Koestner, R. y Ryan, R. M. (2001). Extrinsic rewards and intrinsic motivation in education: Reconsidered once again. Review of Educational Research, 71 (1), 1-27. 
MELCHOR GUTIÉRREZ, JOSÉ MANUEL TOMÁS, JOSÉ MARCOS BARRICA E ISABEL ROMERO

INFLUENCIA DEL CLIMA MOTIVACIONAL EN CLASE SOBRE EL COMPROMISO ESCOLAR DE LOS ADOLESCENTES Y SU LOGRO ACADÉMICO

Deci, E. L. y Ryan, R. M. (1985). Intrinsic motivation and self-determination in buman behavior. New York: Plenum Press.

Deci, E. L. y Ryan, R. M. (2008). Self-determination theory: A machrotheory of human motivation, development, and health. Canadian Psychology, 49 (3), 182-185.

Eurostat (2013). Eurostat regional yearbook 2013. Statistical Book. European Commission. Luxembourg: Publications Office Eu.

Finney, S. J. y DiStefano, C. (2006). Non-normal and categorical data in SEM. En G. R. Hancock y R. O. Mueller (Eds.). Structural equation modelling: A second course (pp. 269314). Greenwich, CO: Information Age Publishing.

Fredricks, J. A.; Blumenfeld, P. C. y Paris, A. H. (2004). School engagement: Potential of the concept, state of the evidence. Review of Educational Research, 74, 59-90.

Froiland, J. M. y Oros, E. (2014). Intrinsic motivation, perceived competence and classroom engagement as longitudinal predictors of adolescent reading achievement. Educational Psychology, 34, 119-132.

Froiland, J. M. y Worrell, F. C. (2016). Intrinsic motivation, learning goals, engagement, and achievement in a diverse high school. Psychology in the Schools, 53 (3), 321-336.

Gordon, M. S. (2016). Community disadvantage and adolescent's academic achievement: the mediating role of father influence. Journal of Child and Family Studies, 25 (7), 2069-2078.

Green, J.; Liem, G. A.; Martin, A. J.; Colmar, S.; Marsh, H. W. y McInerney, D. (2012). Academic motivation, self-concept, engagement, and performance in high school: Key processes from a longitudinal perspective. Journal of Adolescence, 35, 1111-1122.

Greene, B. A.; Miller, R. B.; Crowson, H. M.; Duke, B. L. y Akey, K. L. (2004). Predicting high school students' cognitive engagement and achievement: Contributions of classroom perceptions and motivation. Contemporary Educational Psychology, 29, 462-482.

Hu, L. y Bentler, P. M. (1999). Cutoff criteria for fit indexes in covariance structure analysis: Conventional criteria versus new alternatives. Structural Equation Modeling, 6, 1-55.

Jang, H.; Kim, E. U. y Reeve, J. (2012). Longitudinal test of Self-Determination Theory's motivation mediation model in a naturally occurring classroom context. Journal of Educational Psychology, 104 (4), 1175-1188.

Jelas, Z. M.; Azman, N.; Zulnaidi, H. y Ahmad, N. A. (2016). Learning support and academic achievement among Malaysian adolescents: the mediating role of student engagement. Learning Environments Research, 19 (2), 221-240.

Jeynes, W. H. (2007). The relationship between parental involvement and urban secondary school student academic achievement: A meta-analysis. Urban Education, 42 (1), $82-110$.

Kaplan, D. (2000). Structural equation modeling: Foundations and extensions. Thousand Oaks, CA: Sage.

Lau, S. y Roeser, R. W. (2002). Cognitive abilities and motivational processes in high school students' situational engagement and achievement in science. Educational Assessment, 8 (2), 139-162.

Lee, C. S.; Hayes, K. N.; Seitz, J.; DiStefano, R. y O'Connor, D. (2016). Understanding motivational structures that differentially predict engagement and achievement in middle school science. International Journal of Science Education, 38 (2), 192-215.

Linnenbrink, E. A. y Pintrich, P. R. (2002). Achievement goal theory and affect: An asymmetrical bi-directional model. Educational Psychologist, 37, 69-78. 
MELCHOR GUTIÉRREZ, JOSÉ MANUEL TOMÁS, JOSÉ MARCOS BARRICA E ISABEL ROMERO INFLUENCIA DEL CLIMA MOTIVACIONAL EN CLASE SOBRE EL COMPROMISO ESCOLAR DE LOS ADOLESCENTES Y SU LOGRO ACADÉMICO

Martin, A. J.; Collie, R. J.; Mok, M. M. C. y McInermey, D. M. (2016). Personal best (PB) goal structure, individual PB goals, engagement, and achievement: A study of Chinese-and English-speaking background students in Australian schools. British Journal of Educational Psychology, 86 (1), 75-91.

Mega, C.; Ronconi, L. y De Beni, R. (2014). What makes a good student? How emotions, self-regulated learning, and motivation contribute to academic achievement. Journal of Educational Psychology, 106 (1), 121-131.

Nicholls, J. (1989). The competitive ethos and democratic education. Cambridge, MA: Harvard University Press.

Niemiec, C. P. y Ryan, R. M. (2009). Autonomy, competence, and relatedness in the classroom: Applying self-determination theory to educational practice. Theory and Research in Education, 7, 133-144.

Noble, J. P. y Sawyer, R. L. (2004). Is high school GPA better than admission test scores for predicting academic success in college? College and University, 79 (4), 17-22.

Pintrich, P. R. (2004). A conceptual framework for assessing student motivation and self-regulated learning in college students. Educational Psychology Review, 16, 385-407.

Plunkett, S. W.; Henry, C. S.; Houltberg, B. J.; Sands, T. y Abarca-Mortensen, S. (2008). Academic support by significant others and educational resilience in Mexican-origin ninth grade students from intact families. The Journal of Early Adolescence, 28 (3), 333-355.

Raufelder, D.; Kittler, F.; Braun, S. R.; Lätsch, A.; Wilkiinson, R. P. y Hoferichter, F. (2014). The interplay of perceived stress, self-determination and school engagement in adolescence. School Psychology International, 35 (4), 405-420.

Raufelder, D.; Regner, N.; Drury, K. y Eid, M. (2016). Does self-determination predict the school engagement of four different motivation types in adolescence? Educational Psychology, 36 (7), 1242-1263.

Reeve, J. (2009). Why teachers adopt a controlling motivating style toward students and how they can become more autonomy supportive. Educational Psychologist, 44, 159-175.

Reeve, J. (2012). A self-determination theory perspective on student engagement. En S. L. Christenson, A. L. Reschly y C. Wylie (Eds.). Handbook of research on student engagement. New York: Springer.

Reeve, J. y Halusic, M. (2009). How K-12 teachers can put self-determination theory principles into practice. Theory and Research in Education, 7 (2), 145-154.

Reeve, J.; Jang, H.; Carrell, D.; Jeon, S. y Barch, J. (2004). Enhancing students' engagement by increasing teachers' autonomy support. Motivation and Emotion, 28, 147-169.

Reeve, J. y Tseng, C. (2011). Personal agency as a fourth aspect of students' engagement during learning activities. Contemporary Educational Psychology, 36, 257-267.

Reynolds, D.; Sammons, P.; De Fraine, B.; Damme, J. V.; Townsend, T.; Teddlie, C. y Stringfield, S. (2014). Educational effectiveness research (EER): A state-of-the-art review. School Effectiveness and School Improvement, 25, 197-230.

Ryan, R. M. y Deci, E. L. (2000). Self-determination theory and the facilitation of intrinsic motivation, social development, and well-being. American Psychologist, 55, 68-78.

Ryan, R. M. y Deci, E. L. (2009). Promoting self-determined school engagement: Motivation, learning, and well-being. En K. R. Wentzel y A. Wigfield (Eds.). Handbook of motivation at school (pp. 171-196). New York: Routledge.

Senko, C.; Hulleman, C. S. y Harackiewicz, J. M. (2011). Achievement goal theory at the crossroads: Old controversies, current challenges, and new directions. Educational Psychologist, 46, 26-47. 
MELCHOR GUTIÉRREZ, JOSÉ MANUEL TOMÁS, JOSÉ MARCOS BARRICA E ISABEL ROMERO DE LOS ADOLESCENTES Y SU LOGRO ACADÉMICO

Sheldon, K. M. y Hilper, J. C. (2012). The Balanced Measure of Psychological Needs (BMPN) scale: An alternative domain general measure of need satisfaction. Motivation and Emotion, 36, 439-451.

Skinner, E. A.; Furrer, C.; Marchand, G. y Kindermann, T. (2008). Engagement and disaffection in the classroom: Part of a larger motivational dynamic? Journal of Educational Psychology, 100, 765-781.

Skinner, E. A.; Kindermann, T. A. y Furrer, C. (2009). A motivational perspective on engagement and disaffection: Conceptualization and assessment of children's behavioral and emotional participation in academic activities in the classroom. Educational and Psychological Measurement, 69, 493-525.

Stornes, E. B. y Bru, E. (2011). Perceived motivational climates and self-reported emotional and behavioural problems among Norwegian secondary school students. School Psychology International, 32, 425-438.

Tomás, J. M.; Gutiérrez, M.; Sancho, P.; Chireac, S. M. y Romero, I. (2016). El compromiso escolar (school engagement) de los adolescentes: Medida de sus dimensiones. Enseñanza \& Teaching, 34 (1), 119-135.

Vansteenkiste, M.; Simons, J.; Lens, W.; Sheldon, K. M. y Deci, E. L. (2004). Motivating learning, performance, and persistence: The synergistic role of intrinsic goals and autonomy support. Journal of Personality and Social Psychology, 87, 246-260.

Vansteenkiste, M.; Simons, J.; Lens, W.; Soenens, B. y Matos, L. (2005). Examining the impact of extrinsic versus intrinsic goal framing and internally controlling versus autonomy-supportive communication style upon early adolescents' academic achievement. Child Development, 76, 483-501.

Veiga, F. H. (2013). Envolvimento dos alunos na escola: Elaboração de uma nova escala de avaliação. International Journal of Developmental and Educational Psychology, 1 (1) 441-450.

Wang, M. T. y Holcombe, R. (2010). Adolescents' perceptions of school environment, engagement, and academic achievement in middle school. American Educational Research Journal, 47 (3), 633-662.

Winne, P. H. y Nesbit, J. C. (2010). The psychology of academic achievement. Annual Review of Psychology, 61, 653-678.

Xia, M.; Fosco, G. M. y Feinberg, M. E. (2016). Examining reciprocal influences among family climate, school attachment, and academic self-regulation: implications for school success. Journal of Family Psychology, 30 (4), 442-452. 